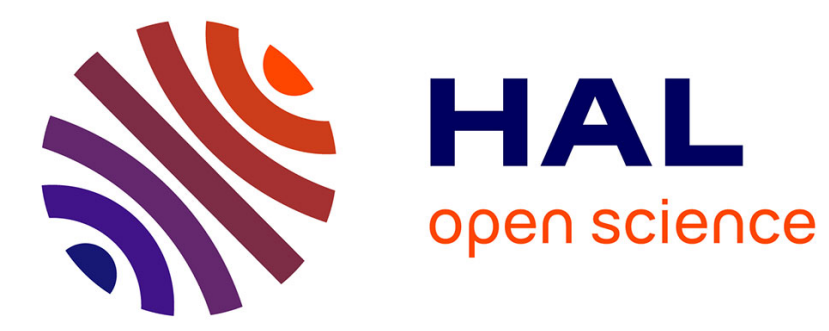

\title{
Asymptotic High-SNR Capacity of MISO Optical Intensity Channels
}

\author{
Stefan M Moser, Ligong Wang, Michèle Wigger
}

\section{To cite this version:}

Stefan M Moser, Ligong Wang, Michèle Wigger. Asymptotic High-SNR Capacity of MISO Optical Intensity Channels. 2017 IEEE Information Theory Workshop (ITW 2017), Nov 2017, Kaohsiung, Taiwan. 10.1109/ITW.2017.8277933 . hal-01700471

\section{HAL Id: hal-01700471 \\ https://hal.science/hal-01700471}

Submitted on 31 Aug 2021

HAL is a multi-disciplinary open access archive for the deposit and dissemination of scientific research documents, whether they are published or not. The documents may come from teaching and research institutions in France or abroad, or from public or private research centers.
L'archive ouverte pluridisciplinaire HAL, est destinée au dépôt et à la diffusion de documents scientifiques de niveau recherche, publiés ou non, émanant des établissements d'enseignement et de recherche français ou étrangers, des laboratoires publics ou privés. 


\title{
Asymptotic High-SNR Capacity of MISO Optical Intensity Channels
}

\author{
Stefan M. Moser*†, Ligong Wang ${ }^{\ddagger}$, and Michèle Wigger ${ }^{\S}$ \\ ${ }^{*}$ Signal and Information Processing Lab, ETH Zürich, Switzerland \\ ${ }^{\dagger}$ Department of Electrical and Computer Engineering, National Chiao Tung University, Hsinchu, Taiwan \\ ‡ETIS-Université Paris Seine, Université de Cergy-Pontoise, ENSEA, CNRS, Cergy-Pontoise, France \\ ${ }^{\S}$ LTCI, Telecom ParisTech, Université Paris-Saclay, 75013 Paris, France
}

\begin{abstract}
This paper derives the asymptotic capacity for the multiple-input single-output free-space optical intensity channel in the regime of high signal-to-noise ratio (SNR). The asymptotic result is proven via upper and lower bounds on capacity at finite SNR.
\end{abstract}

\section{INTRODUCTION}

Optical wireless communication is a form of communication in which visible, infrared, or ultraviolet light is transmitted in free space (air or vacuum) to carry a message to its destination. Recent works suggest that it is a promising solution to replacing some of the existing radio-frequency (RF) wireless communication systems in order to prevent future rate bottlenecks [1]-[3]. Particularly attractive are simple intensity-modulation-direct-detection (IM-DD) systems. In such a system, the transmitter modulates the intensity of optical signals coming from light emitting diodes (LEDs) or laser diodes (LDs), and the receiver measures incoming optical intensities by means of photodetectors. The electrical output signals of the photodetectors are essentially proportional to the incoming optical intensities, but are corrupted by thermal noise of the photodetectors, relative-intensity noise of random intensity fluctuations inherent to low-cost LEDs and LDs, and shot noise caused by ambient light. In a first approximation, noise coming from these sources is usually modeled as being additive Gaussian and independent of the transmitted light signal; see [1], [2].

The free-space optical intensity channel has been extensively studied in the literature. In the single-input singleoutput (SISO) scenario, where the transmitter employs a single transmit LED or LD, and the receiver a single photodetector, the works [4], [5] established upper and lower bounds on the capacity of this channel that are asymptotically tight in both high-signal-to-noise-ratio (high-SNR) and low-SNR limits. Improved bounds at finite SNR have subsequently been presented in [6]-[9]. For the multiple-input and multipleoutput (MIMO) optical intensity channel, where the transmitter is equipped with multiple LEDs or LDs, and the receiver with multiple photodetectors, the recent work [10] has derived several asymptotic capacity results in the high-SNR limit; see details below. Previous to [10], various code constructions for this setup have been proposed in [11]-[14]. When there is no crosstalk so the MIMO channel can be modeled through a diagonal channel matrix, bounds on capacity were presented in [9], [15].

The main contributions of [10] are the asymptotic high-SNR capacity for the MIMO channel in the following cases:

- The channel matrix is of full column rank, i.e., its rank equals the number of transmit LEDs, and the inputs are subject to any peak- and average-power constraints;

- The channel is multiple-input and single-output (MISO), and the inputs are subject to only a peak-power constraint, or only an average-power constraint, or both constraints but with the average-power constraint being sufficiently loose.

In the current work, we consider the MISO channel in the regime where there are both average- and peak-power constraints, and where the average-power constraint is not "sufficiently loose." Together with the MISO results in [10], this completely characterizes the asymptotic high-SNR capacity of any MISO channel for all parameter ranges.

The basic tools for proving our new result are similar to those used in [5], [9], [10]: our capacity lower bound is derived using the Entropy Power Inequality (EPI), and our upper bound is based on duality [16]. However, the proofs are much more involved. On the one hand, the optimal input distribution for the lower bound turns out to be more complex than those in [5], [10]: it involves LED cooperation (compared to independent signaling in the MIMO full-columnrank case [10]), and, with certain probabilities, assigns to each LED a truncated exponential distribution (compared to either exponential or uniform distribution in the special MISO cases in [10]), whose parameters must be optimized. On the other hand, the output distribution chosen in the duality-based upper bound depends on the capacity-achieving input distribution, and its analysis is based on a new lemma that bounds the capacity-achieving probability measure on a given interval.

\section{Channel Model and Main Result}

\section{A. Channel Model}

Consider a communication link where the transmitter is equipped with $n_{\mathrm{T}}$ LEDs (or LDs) and the receiver with a single photodetector. The photodetector receives a superposition of the signals sent by the LEDs, and we assume that the crosstalk 
between LEDs is constant. Hence, the channel output is given by

$$
Y=\mathbf{h}^{\top} \mathbf{x}+Z
$$

where the $n_{\mathrm{T}}$-vector $\mathbf{x}$ denotes the channel input, whose entries are proportional to the optical intensities of the corresponding LEDs, and are therefore nonnegative:

$$
x_{i} \in \mathbb{R}_{0}^{+}, \quad i=1, \ldots, n_{\mathrm{T}} ;
$$

where the length- $n_{\mathrm{T}}$ row vector $\mathbf{h}^{\top}$ is the constant channel state vector with nonnegative entries, which, without loss of generality, we assume to be ordered:

$$
h_{1} \geq h_{2} \geq \cdots \geq h_{n_{\mathrm{T}}}>0
$$

and where $Z \sim \mathcal{N}\left(0, \sigma^{2}\right)$ is additive Gaussian noise that is independent of $\mathbf{X}$. Note that, in contrast to the input $\mathbf{x}$, the output $Y$ can be negative.

Inputs are subject to a peak-power (peak-intensity) and an average-power (average-intensity) constraint:

$$
\begin{gathered}
\operatorname{Pr}\left[X_{i}>\mathrm{A}\right]=0, \quad \forall i \in\left\{1, \ldots, n_{\mathrm{T}}\right\}, \\
\sum_{i=1}^{n_{\mathrm{T}}} \mathrm{E}\left[X_{i}\right] \leq \mathrm{E}
\end{gathered}
$$

for some fixed parameters $A, E>0$. Note that the averagepower constraint is on the expectation of the channel input and not on its square. Also note that $A$ describes the maximum power of each single LED, while $E$ describes the allowed average total power of all LEDs together.

We denote the ratio between the allowed average power and the allowed peak power by $\alpha$ :

$$
\alpha \triangleq \frac{E}{A},
$$

where $0<\alpha \leq n_{\mathrm{T}}$. For $\alpha=n_{\mathrm{T}}$ the average-power constraint is inactive in the sense that it is automatically satisfied whenever the peak-power constraint is satisfied. Thus, $\alpha=n_{\mathrm{T}}$ corresponds to the case with only a peak-power constraint. On the other hand, $\alpha \ll 1$ corresponds to having a dominant average-power constraint and only a very weak peak-power constraint.

We denote the capacity of the channel (1) with allowed peak power $A$ and allowed average power $E$ by $C_{\mathbf{h}^{\top}, \sigma^{2}}(A, E)$. The capacity is given by [17]

$$
\mathrm{C}_{\mathbf{h}^{\top}, \sigma^{2}}(\mathrm{~A}, \mathrm{E})=\sup _{Q} \mathrm{I}(Q, W)
$$

where the supremum is over all laws $Q$ on $\mathbf{X}$ satisfying (2), (4), and (5). When only an average-power constraint is imposed, capacity is denoted by $\mathrm{C}_{\mathbf{h}^{\top}, \sigma^{2}}(\mathrm{E})$. It is given as in (7) except that the supremum is taken over all laws $Q$ on $\mathbf{X}$ satisfying (2) and (5).

\section{B. Previous Results}

Denote

$$
\bar{X} \triangleq \mathbf{h}^{\top} \mathbf{X}
$$

Because $\mathbf{X} \multimap \bar{X} \multimap Y$ form a Markov chain, and because $\bar{X}$ is a function of $\mathbf{X}$, we have [10]

$$
\mathrm{I}(\mathbf{X} ; Y)=\mathrm{I}(\bar{X} ; Y) .
$$

Hence the MISO channel (1) is equivalent to the SISO channel whose input is $\bar{X}$, while the constraints (4) and (5) on $\mathbf{X}$ are transformed to a set of admissible distributions for $\bar{X}$.

Define the following quantities:

$$
\begin{aligned}
h_{\text {sum }} & \triangleq \sum_{i=1}^{n_{\mathrm{T}}} h_{i} \\
\alpha_{\text {th }} & \triangleq \frac{1}{2}+\frac{1}{h_{\text {sum }}} \sum_{k=1}^{n_{\mathrm{T}}} h_{k}(k-1) .
\end{aligned}
$$

In the case where there is no peak-power constraint, or where $\alpha \geq \alpha_{\text {th }}$, the asymptotic high-SNR capacity of the above MISO channel is given by [10, Cor. $7 \&$ Thm. 8]:

Theorem 1 ([10]): When $\alpha \geq \alpha_{\mathrm{th}}$,

$$
\lim _{A \rightarrow \infty}\left\{C_{\mathbf{h}^{\top}, \sigma^{2}}(A, \alpha A)-\log A\right\}=\frac{1}{2} \log \frac{h_{\text {sum }}^{2}}{2 \pi e \sigma^{2}} .
$$

Without a peak-power constraint,

$$
\lim _{\mathrm{E} \rightarrow \infty}\left\{\mathrm{C}_{\mathbf{h}^{\top}, \sigma^{2}}(\mathrm{E})-\log \mathrm{E}\right\}=\frac{1}{2} \log \frac{e h_{1}^{2}}{2 \pi \sigma^{2}} .
$$

\section{Main Result}

The main result of this paper characterizes the asymptotic high-SNR capacity of the MISO channel in the regime that is not covered by Theorem 1 .

Theorem 2: If $\alpha<\alpha_{\mathrm{th}}$, then

$$
\begin{aligned}
& \lim _{A \rightarrow \infty}\left\{C_{\mathbf{h}^{\top}, \sigma^{2}}(A, \alpha A)-\log A\right\} \\
& =\frac{1}{2} \log \frac{h_{\mathrm{sum}}^{2}}{2 \pi e \sigma^{2}}-\inf _{\lambda \in\left(0, \min \left\{\frac{1}{2}, \alpha\right\}\right)}\left\{\log \frac{\mu(\lambda)}{1-e^{-\mu(\lambda)}}-1\right. \\
& \left.+\frac{\mu(\lambda) e^{-\mu(\lambda)}}{1-e^{-\mu(\lambda)}}+\operatorname{pinf}_{\mathbf{p}: \sum_{k=1}^{n_{\mathrm{T}}} p_{k}(k-1)=\alpha-\lambda} \mathscr{D}\left(\mathbf{p} \| \frac{\mathbf{h}}{h_{\mathrm{sum}}}\right)\right\},
\end{aligned}
$$

where $\mathbf{p}$ denotes a probability vector, i.e., $p_{1}, \ldots, p_{n_{\mathrm{T}}}$ are nonnegative and sum to one; where $\mathscr{D}(\mathbf{p} \| \mathbf{q})$ denotes relative entropy between the probability vectors $\mathbf{p}$ and $\mathbf{q}$ :

$$
\mathscr{D}(\mathbf{p} \| \mathbf{q}) \triangleq \sum_{k=1}^{n_{\mathrm{T}}} p_{k} \log \frac{p_{k}}{q_{k}} ;
$$

and where $\mu(\lambda)$ is the unique positive solution for $\mu$ to

$$
\frac{1}{\mu}-\frac{e^{-\mu}}{1-e^{-\mu}}=\lambda \text {. }
$$

Theorem 2 is proven via lower and upper bounds on the capacity. The proof will be outlined in Section III. Before doing so, we make some remarks about this result 


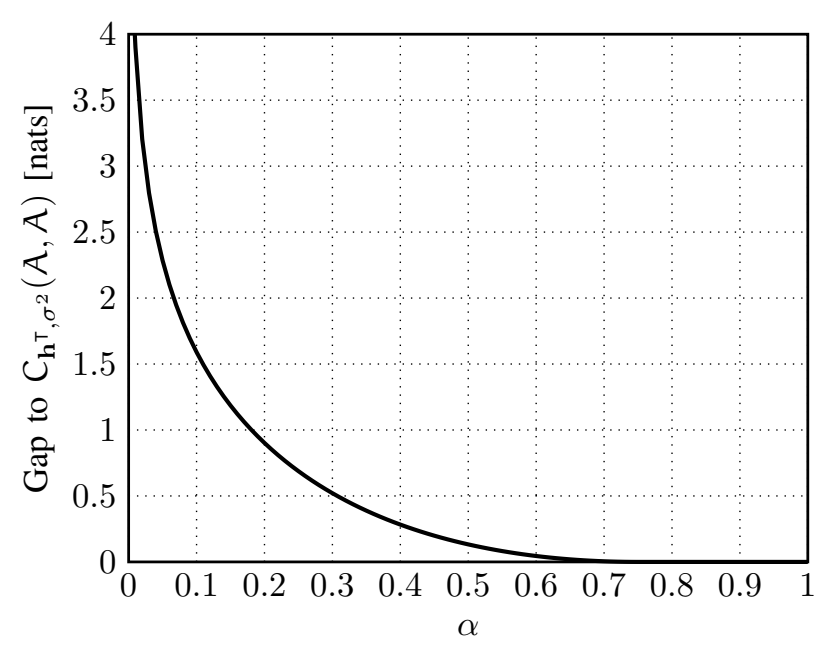

Fig. 1. The outer infimum in (14) as a function of $\alpha$, for MISO channel with two transmit LEDs, $h_{1}=3$ and $h_{2}=1$. This infimum is the asymptotic capacity gap to the case with no average-power constraint $C_{\mathbf{h}^{\top}, \sigma^{2}}(A, A)$.

Optimization in (14). For a fixed $\lambda$, the optimal choice for $\mathbf{p}=\left(p_{1}, \ldots, p_{k}\right)$ is (see, e.g., [18, Problem 12.2])

$$
p_{k}^{*}=\frac{h_{k} a^{k}}{\sum_{j=1}^{n_{\mathrm{T}}} h_{j} a^{j}}, \quad k=1, \ldots, n_{\mathrm{T}},
$$

where $a \geq 0$ is obtained by solving the following equation:

$$
\frac{\sum_{k=1}^{n_{\mathrm{T}}} h_{k} k a^{k}}{\sum_{j=1}^{n_{\mathrm{T}}} h_{j} a^{j}}=\alpha-\lambda+1 .
$$

The optimization over $\lambda$ can easily be performed numerically.

Asymptotically Optimal Input Distributions. As our capacity lower bound will show, in the high-SNR limit, the optimal strategy in terms of capacity is to maximize the differential entropy $h(\bar{X})$. One can show that the optimal distribution for $\mathbf{X}$ in this sense is given by the following: with probability $p_{k}$, $k \in\left\{1, \ldots, n_{\mathrm{T}}\right\}$,

$$
\begin{aligned}
& X_{j}=A \quad \text { w.p. } 1, \quad j \in\{1, \ldots, k-1\}, \\
& X_{k} \sim \text { truncated exponential of parameter } \mu, \\
& X_{j}=0 \quad \text { w.p. } 1, \quad j \in\left\{k+1, \ldots, n_{\mathrm{T}}\right\},
\end{aligned}
$$

where $\mathbf{p}$ and $\mu$ are the optimal choices of $\mathbf{p}$ and $\mu(\lambda)$, respectively, in (14). Note that, when $\alpha \geq \alpha_{\mathrm{th}}$, [10] shows that the truncated exponential distribution in (19b) should be replaced by a uniform distribution, and the optimal $\mathbf{p}$ is given by $p_{k}=h_{k} / h_{\text {sum }}, k \in\left\{1, \ldots, n_{\mathrm{T}}\right\}$. These choices yield a uniform distribution on $\left[0, h_{\text {sum }} A\right]$ for $\bar{X}$. When $\alpha<\alpha_{\text {th }}$, it is no longer possible to achieve a uniform distribution for $\bar{X}$ without violating the average-power constraint (5). Instead, the optimal distribution for $\bar{X}$ is a concatenation of $n_{\mathrm{T}}$ truncated exponentials.

An Example. Consider a MISO channel with two transmit LEDs and with channel parameters $h_{1}=3$ and $h_{2}=1$. We plot the outer infimum in (14) against $\alpha$ in Fig. 1. Note that this infimum characterizes the capacity gap to the case with no average-power constraint, in the high-SNR limit. As expected, the gap becomes zero when $\alpha$ reaches $\alpha_{\text {th }}=0.75$, and approaches infinity when $\alpha$ tends to zero.

\section{PRoOF OUTLINE}

\section{A. Achievability: Lower Bound}

We prove the following lower bound on capacity, from which the achievability part of Theorem 2 follows by optimization over the parameters.

Proposition 3: Assume $\alpha<\alpha_{\mathrm{th}}$. For any probability vector p such that

$$
0<\alpha-\sum_{k=1}^{n_{\mathrm{T}}} p_{k}(k-1)<\frac{1}{2}
$$

we have

$$
\begin{aligned}
\mathrm{C}_{\mathbf{h}^{\top}, \sigma^{2}}(\mathrm{~A}, \alpha \mathrm{A}) \geq & \log \mathrm{A}+\frac{1}{2} \log \frac{h_{\mathrm{sum}}^{2}}{2 \pi e \sigma^{2}}-\log \frac{\mu^{*}}{1-e^{-\mu^{*}}} \\
& +1-\frac{\mu^{*} e^{-\mu^{*}}}{1-e^{-\mu^{*}}}-\mathscr{D}\left(\mathbf{p} \| \frac{\mathbf{h}}{h_{\text {sum }}}\right)
\end{aligned}
$$

where $\mu^{*}$ is the unique positive solution to

$$
\frac{1}{\mu^{*}}-\frac{e^{-\mu^{*}}}{1-e^{-\mu^{*}}}=\alpha-\sum_{k=1}^{n_{\mathrm{T}}} p_{k}(k-1) .
$$

Proof: Choosing a particular input distribution (specified below) and using the Entropy Power Inequality (EPI) [18, Thm. 17.7.3] we obtain

$$
\begin{aligned}
\mathrm{C}_{\mathbf{h}^{\top}, \sigma^{2}}(\mathrm{~A}, \alpha A) \\
\quad \geq \mathrm{I}(\bar{X} ; \bar{X}+Z) \\
\quad=\mathrm{h}(\bar{X}+Z)-\mathrm{h}(Z) \\
\quad \geq \frac{1}{2} \log \left(e^{2 \mathrm{~h}(\bar{X})}+e^{2 \mathrm{~h}(Z)}\right)-\mathrm{h}(Z) \\
\quad=\mathrm{h}(\bar{X})-\frac{1}{2} \log 2 \pi e \sigma^{2}+\frac{1}{2} \log \left(1+\frac{2 \pi e \sigma^{2}}{e^{2 \mathrm{~h}(\bar{X})}}\right) \\
\quad \geq \mathrm{h}(\bar{X})-\frac{1}{2} \log 2 \pi e \sigma^{2} .
\end{aligned}
$$

Now we fix some probabilities $p_{1}, \ldots, p_{n_{\mathrm{T}}}$ summing to one and satisfying (20), and we define a random variable (RV) $U \in\left\{1, \ldots, n_{\mathrm{T}}\right\}$ with $\operatorname{Pr}[U=k]=p_{k}$. Conditional on $U=$ $k$, we choose

$$
\left.X_{j}\right|_{\text {cond. on } U=k}= \begin{cases}\text { A } & \text { if } j=1, \ldots, k-1, \\ 0 & \text { if } j=k+1, \ldots, n_{\mathrm{T}}\end{cases}
$$

and we choose $X_{k}$ to be distributed according to the law

$$
\left.X_{k}\right|_{\text {cond. on } U=k} \sim \frac{1}{\mathrm{~A}} \frac{\mu^{*}}{1-e^{-\mu^{*}}} e^{-\frac{\mu^{*} x}{\mathrm{~A}}} \mathbf{I}\{0<x \leq \mathrm{A}\},
$$

where $\mu^{*}$ is the unique positive solution to $(22)$. Note that

$$
\begin{aligned}
\mathrm{E}\left[\sum_{j=1}^{n_{\mathrm{T}}} X_{j}\right] & =\sum_{k=1}^{n_{\mathrm{T}}} p_{k} \sum_{j=1}^{n_{\mathrm{T}}} \mathrm{E}\left[X_{j} \mid U=k\right] \\
& =\sum_{k=1}^{n_{\mathrm{T}}} p_{k}\left((k-1) \mathrm{A}+\left(\frac{1}{\mu^{*}}-\frac{e^{-\mu^{*}}}{1-e^{-\mu^{*}}}\right) \mathrm{A}\right)
\end{aligned}
$$




$$
=\alpha A,
$$

where the last equality holds because of (22). Thus, this choice of input satisfies both (4) and (5). Also note that conditional on $U$, only one antenna has a random input, while the others are deterministic. Therefore it is possible to deduce from $\bar{X}$ the value of $U$ :

$$
U=k \Longleftrightarrow \bar{X} \in\left(\sum_{j=1}^{k-1} h_{j}, \sum_{j=1}^{k} h_{j}\right] .
$$

Thus,

$$
\begin{aligned}
\mathrm{h}(\bar{X})= & \mathrm{h}(\bar{X})-\mathrm{h}(\bar{X} \mid U)+\mathrm{h}(\bar{X} \mid U) \\
= & \mathrm{I}(U ; \bar{X})+\mathrm{h}(\bar{X} \mid U) \\
= & \mathrm{H}(U)-\underbrace{\mathrm{H}(U \mid \bar{X})}_{=0}+\mathrm{h}(\bar{X} \mid U) \\
= & \mathrm{H}(\mathbf{p})+\sum_{k=1}^{n_{\mathrm{T}}} p_{k} \mathrm{~h}(\bar{X} \mid U=k) \\
= & \mathrm{H}(\mathbf{p})+\sum_{k=1}^{n_{\mathrm{T}}} p_{k} \mathrm{~h}\left(h_{k} X_{k} \mid U=k\right) \\
= & \mathrm{H}(\mathbf{p})+\sum_{k=1}^{n_{\mathrm{T}}} p_{k} \log h_{k}+\log A-\log \frac{\mu^{*}}{1-e^{-\mu^{*}}} \\
& +1-\frac{\mu^{*} e^{-\mu^{*}}}{1-e^{-\mu^{*}}},
\end{aligned}
$$

where in (38) we use that conditional on $U=k$, only $X_{k}$ is random, the other RVs are deterministic and can therefore be subtracted from $\bar{X}$.

Combining (39) with (27) now proves the claim.

\section{B. Converse: Upper Bound}

We first derive the following upper bound on capacity.

Proposition 4: For every $k \in\left\{0, \ldots, n_{\mathrm{T}}\right\}$, define

$$
s_{k} \triangleq \sum_{j=1}^{k} h_{j}
$$

Let $p_{k}, k \in\left\{2, \ldots, n_{\mathrm{T}}\right\}$, denote the probability that $\bar{X} \in$ $\left(s_{k-1} \mathrm{~A}, s_{k} \mathrm{~A}\right]$, and $p_{1}$ that $\bar{X} \in\left[0, s_{1} \mathrm{~A}\right]$, under the capacityachieving input distribution. For any choice of parameters $\mu, \delta, \eta \in(0, \infty), \beta \in\left(0, h_{n_{\mathrm{T}}}\right)$, and $n_{\mathrm{T}}$-dimensional probability vector $\mathbf{q}$, we have

$$
\begin{aligned}
& \mathrm{C}_{\mathbf{h}^{\top}, \sigma^{2}}(\mathrm{~A}, \alpha \mathrm{A}) \\
& \leq \max \left\{1, \log 2 \pi e \sigma^{2}\right\} \cdot \mathcal{Q}\left(\frac{\delta \mathrm{A}}{\sigma}\right)+\frac{\delta \mathrm{A}}{\sqrt{2 \pi} \sigma} e^{-\frac{\delta^{2} \mathrm{~A}^{2}}{2 \sigma^{2}}} \\
& \quad-\log \left(1-2 \mathcal{Q}\left(\frac{\delta \mathrm{A}}{\sigma}\right)-2\left(n_{\mathrm{T}}-1\right) \frac{\delta \mathrm{A}}{\eta}\right) \\
& +\log \eta \sum_{k=1}^{n_{\mathrm{T}}}\left(\mathcal{Q}\left(\frac{\left(h_{k}-\delta\right) \mathrm{A}}{\sigma}\right)-\mathcal{Q}\left(\frac{\left(h_{k}+\delta\right) \mathrm{A}}{\sigma}\right)\right. \\
& \left.+\mathcal{Q}\left(\frac{\left(h_{k+1}-\delta\right) \mathcal{A}}{\sigma}\right)-\mathcal{Q}\left(\frac{\left(h_{k+1}+\delta\right) \mathcal{A}}{\sigma}\right)\right)
\end{aligned}
$$

$$
\begin{aligned}
& +2 n_{\mathrm{T}} \log \eta \cdot \frac{\log 2+\frac{1}{e}+\frac{1}{2} \log \left(1+\frac{\beta^{2} \mathrm{~A}^{2}}{4 \sigma^{2}}\right)}{\frac{1}{2} \log \left(1+\frac{\mathrm{A}^{2}}{2 \pi e \sigma^{2}} \cdot \Upsilon(\alpha, \delta)\right)} \\
& +2 \log \eta \cdot\left(\mathcal{Q}\left(\frac{(\beta-\delta) \mathrm{A}}{\sigma}\right)-\mathcal{Q}\left(\frac{(\beta+\delta) \mathrm{A}}{\sigma}\right)\right) \\
& +\mathcal{Q}\left(\frac{\delta \mathrm{A}}{\sigma}\right) \\
& +\sum_{k=1}^{n_{\mathrm{T}}} \max \left\{0, \log \left(\frac{\mathrm{A}\left(h_{k}-2 \delta\right)}{1-2 \mathcal{Q}\left(\frac{\delta \mathrm{A}}{\sigma}\right)-2\left(n_{\mathrm{T}}-1\right) \frac{\delta \mathrm{A}}{\eta}}\right)\right\} \\
& +\log \mathrm{A}-\frac{1}{2} \log 2 \pi e \sigma^{2}+\mathcal{Q}\left(\frac{\delta \mathrm{A}}{\sigma}\right) \sum_{k=1}^{n_{\mathrm{T}}}\left(\frac{\mu s_{n_{\mathrm{T}}}^{h_{k}}-\log q_{k}}{+}\right) \\
& \frac{\mu \sigma}{\mathrm{A} \sqrt{2 \pi}} \sum_{k=1}^{n_{\mathrm{T}}} \frac{1}{h_{k}}+\sum_{k=1}^{n_{\mathrm{T}}} p_{k} \log \frac{h_{k}}{q_{k}}-\log \mu \\
& +\log \left(1-e^{-\mu}\right)+\mu\left(\alpha-\sum_{k=1}^{n_{\mathrm{T}}} p_{k}(k-1)\right),
\end{aligned}
$$

where

$$
\Upsilon(\alpha, \delta) \triangleq \begin{cases}e^{2 \alpha \delta}\left(\frac{1-e^{-\delta}}{\delta}\right)^{2} & \text { if } \alpha \in\left(0, \frac{1}{2}\right), \\ 1 & \text { if } \alpha \geq \frac{1}{2} .\end{cases}
$$

Proof Outline: We apply the duality-based bounding technique [16]

$$
\begin{aligned}
\mathrm{C}_{\mathbf{h}^{\top}, \sigma^{2}}(\mathrm{~A}, \alpha \mathrm{A}) \leq & \mathrm{E}_{Q^{*}}\left[-\int_{-\infty}^{\infty} \frac{1}{\sqrt{2 \pi} \sigma} e^{-\frac{(y-\bar{x})^{2}}{2 \sigma^{2}}} \log f(y) \mathrm{d} y\right] \\
& -\frac{1}{2} \log 2 \pi e \sigma^{2},
\end{aligned}
$$

where $Q^{*}$ is the capacity-achieving input distribution, and $f$ is any probability density function on the real line. Here we define the parameters

$$
\begin{array}{rlrl}
\bar{s}_{k} & \triangleq s_{k}+\delta, & k & =1, \ldots, n_{\mathrm{T}}-1 ; \\
\underline{s}_{k} & \triangleq s_{k}-\delta, & k & =1, \ldots, n_{\mathrm{T}}-1 ; \\
\bar{s}_{0} & \triangleq s_{0}-\delta ; & \\
\underline{s}_{n_{\mathrm{T}}} & \triangleq s_{n_{\mathrm{T}}}+\delta ; & \\
\mu_{k} & \triangleq \frac{\mu}{h_{k}}, & k &
\end{array}
$$

and choose

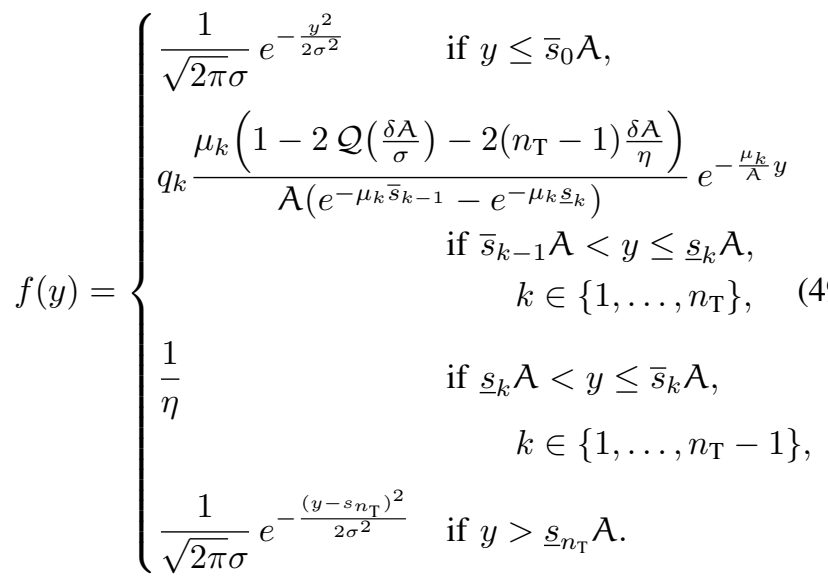


Note that, on the interval $\left[0, h_{\text {sum }} A\right]$, our choice of $f$ resembles the asymptotically optimal distribution for $\bar{X}$, the latter being a concatenation of truncated exponential distributions. But, for technical reasons, we add "buffers" between neighboring truncated exponentials, as well as Gaussian tails on both sides of the interval.

Calculations are omitted due to space limitations.

To use Proposition 4 to derive the desired asymptotic upper bound, we choose

$$
q_{k}=\frac{\mathrm{A} p_{k}+1}{\mathrm{~A}+n_{\mathrm{T}}}, \quad k \in\left\{1, \ldots, n_{\mathrm{T}}\right\} .
$$

Also, denote $\lambda(\mathbf{p}) \triangleq \alpha-\sum_{k=1}^{n_{\mathrm{T}}} p_{k}(k-1)$, fix some $\zeta \in(0,1)$, and choose

$$
\mu= \begin{cases}\mu(\mathbf{p}) & \text { if } A^{-(1-\zeta)}<\lambda(\mathbf{p})<\frac{1}{2}, \\ A^{1-\zeta} & \text { if } \lambda(\mathbf{p}) \leq A^{-(1-\zeta)}, \\ \frac{1}{A} & \text { if } \lambda(\mathbf{p}) \geq \frac{1}{2}\end{cases}
$$

where $\mu(\mathbf{p})$ is the unique positive solution for $\mu$ to

$$
\frac{1}{\mu}-\frac{e^{-\mu}}{1-e^{-\mu}}=\lambda(\mathbf{p})
$$

We then choose

$\delta=\frac{\log (1+A)}{A}, \quad \beta=\frac{\log ^{2}(1+A)}{A}, \eta=\log ^{2}(1+A)$,

and let $A \rightarrow \infty$. After some further calculations, which are again omitted, we obtain that the right-hand side of (14) is an upper bound on its left-hand side.

\section{CONCluding REMARKS}

We have shown that, in the high-SNR limit, the capacity of the peak- and average-intensity limited optical intensity channel is given by the maximum value of $h\left(\mathbf{h}^{\top} \mathbf{X}\right)$ minus the differential entropy of the Gaussian noise. There may be alternative ways to show this asymptotic result. For example, if one can show that, at high SNR, the optimal input distribution has a bounded density, then one may be able to come to the above conclusion via convergence properties of the differential entropy; see, e.g., [19]. On the other hand, such an approach, if successful, would not provide finite-SNR bounds on capacity as our Propositions 3 and 4.

We have also identified the input distribution that maximizes $h\left(\mathbf{h}^{\top} \mathbf{X}\right)$. This input distribution has the following structure: whenever a weaker transmit LED is switched on, all the stronger transmit LEDs must be transmitting at maximum intensity with probability one. Furthermore, conditional on the transmit signal of a specific LED being "random," this signal has a truncated exponential distribution, with a parameter that is the same for all transmit LEDs.

Recall that [10] has established the high-SNR asymptotic capacity of the MIMO optical intensity channel when the channel matrix has full column rank. A close look at the results in [10] confirms that, in the full-column-rank case, the asymptotic capacity is also given by the maximum differential entropy of $\mathrm{HX}, \mathrm{H}$ being the channel matrix, minus that of the noise vector.

With the current work and [10], the only MIMO optical intensity channels whose asymptotic high-SNR capacities are not yet known are those with more than one receive antennas (photodetectors), and with channel matrices that do not have full column rank. It is natural to conjecture that, for those channels, the asymptotic high-SNR capacity is again given by the maximum of $h(H X)$ minus the differential entropy of the noise.

\section{REFERENCES}

[1] J. M. Kahn and J. R. Barry, "Wireless infrared communications," Proc. IEEE, vol. 85, no. 2, pp. 265-298, Feb. 1997.

[2] S. Hranilovic, Wireless Optical Communication Systems. New York, NY, USA: Springer Verlag, 2005.

[3] M. A. Khalighi and M. Uysal, "Survey on free space optical communication: A communication theory perspective," IEEE Comm. Surveys \& Tutorials, vol. 16, no. 4, pp. 2231-2258, fourth quarter 2014.

[4] M. A. Wigger, "Bounds on the capacity of free-space optical intensity channels," Master's thesis, Signal and Inf. Proc. Lab., ETH Zürich, Switzerland, Mar. 2003, supervised by Prof. Dr. Amos Lapidoth.

[5] A. Lapidoth, S. M. Moser, and M. A. Wigger, "On the capacity of free-space optical intensity channels," IEEE Trans. Inf. Theory, vol. 55, no. 10 , pp. 4449-4461, Oct. 2009.

[6] A. L. McKellips, "Simple tight bounds on capacity for the peak-limited discrete-time channel," in Proc. IEEE Int. Symp. Inf. Theory, Chicago, IL, USA, Jun. 27 - Jul. 2, 2004, p. 348.

[7] A. A. Farid and S. Hranilovic, "Channel capacity and non-uniform signalling for free-space optical intensity channels," IEEE J. Select. Areas Commun., vol. 27, no. 9, pp. 1553-1563, Dec. 2009.

[8] — "Capacity bounds for wireless optical intensity channels with Gaussian noise," IEEE Trans. Inf. Theory, vol. 56, no. 12, pp. 60666077, Dec. 2010.

[9] A. Thangaraj, G. Kramer, and G. Böcherer, "Capacity bounds for discrete-time, amplitude-constrained, additive white Gaussian noise channels," arXiv, Nov. 2015, arXiv:1511.08742.

[10] S. M. Moser, M. Mylonakis, L. Wang, and M. Wigger, "Asymptotic capacity results for MIMO wireless optical communication," in Proc. IEEE Int. Symp. Inf. Theory, Aachen, Germany, Jun. 25-30, 2017, pp. 536-540.

[11] S. M. Haas, J. H. Shapiro, and V. Tarokh, "Space-time codes for wireless optical communications," EURASIP J. App. Sig. Proc., no. 3, pp. 211$220,2002$.

[12] E. Bayaki and R. Schober, "On space-time coding for free-space optical systems," IEEE Trans. Commun., vol. 58, no. 1, pp. 58-62, Jan. 2010.

[13] X. Song and J. Cheng, "Subcarrier intensity modulated MIMO optical communications in atmospheric turbulence," IEEE/OSA J. of Optical Comm. \& Networking, vol. 5, no. 9, pp. 1001-1009, Sept. 2013.

[14] L. Mroueh and J.-C. Belfiore, "Quadratic extension field codes for free space optical intensity communications," 2017, to app. in IEEE Trans. Commun.

[15] A. Chaaban, Z. Rezki, and M.-S. Alouini, "Fundamental limits of parallel optical wireless channels: capacity results and outage formulation," IEEE Trans. Commun., vol. 65, no. 1, pp. 296-311, Jan. 2017.

[16] A. Lapidoth and S. M. Moser, "Capacity bounds via duality with applications to multiple-antenna systems on flat fading channels," IEEE Trans. Inf. Theory, vol. 49, no. 10, pp. 2426-2467, Oct. 2003.

[17] C. E. Shannon, "A mathematical theory of communication," Bell Syst. Tech. J., vol. 27, pp. 379-423 and 623-656, Jul. and Oct. 1948.

[18] T. M. Cover and J. A. Thomas, Elements of Information Theory, 2nd ed. New York, NY, USA: Wiley, 2006.

[19] M. Godavarti and A. O. Hero-III, "Convergence of differential entropies," IEEE Trans. Inf. Theory, vol. 50, no. 1, pp. 171-176, Jan. 2004. 